\title{
Retinoic Acid Syndrome, CTCAE
}

National Cancer Institute

\section{Source}

National Cancer Institute. Retinoic Acid Syndrome, CT CAE. NCI Thesaurus. Code C56635.

A disorder characterized by weight gain, dyspnea, pleural and pericardial effusions,

leukocytosis and/or renal failure originally described in patients treated with all-trans retinoic acid. 